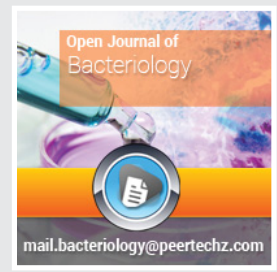

\title{
Open Journal of Bacteriology
}

Mingyue Wang, Miaomiao Yin, Hongming Tan, and Lixiang Cao*

School of Life Sciences, Sun Yat-sen University, Guangzhou, 510275, China

Dates: Received: 30 Decmber, 2016; Accepted: 20 January, 2017; Published: 23 January, 2017

*Corresponding author: Lixiang Cao, School of Life Sciences, Sun Yat-Sen University, Guangzhou, 510275, China, Tel: 86- 20-841 10238, Fax: 86- 2084036215; E-mail: Issclx@163.com

Keywords: Crucian carp; Intestinal flora; Microbiome; Mycobiome

https://www.peertechz.com
Research Article

Illumina Based Analysis of Bacterial and Fungal Flora in Foreguts and Hindguts of Crucian Carp (Carassius Aumtus) in Retail Markets

\section{Introduction}

As with good survival rate, high reproduction rate, and disease resistance, crucian carp (Carassius auratus) is widely bred across Eurasia and America [1]. In China, crucian carp is one of the most economically important freshwater-cultured fish species. The production yield reached nearly 2,000,000 tons in 2009 [2]. However, fish are more perishable than other muscle foods, and a considerable number of fish are spoiled due to lack of good preservation. In world, 30\% of landed fish are spoiled and lost [3].

Fresh fish spoilage can be very rapid after it is caught [3]. Crucian carp is an easily perishable product because of its relatively high quantities of volatile basic nitrogen as well as free amino acids, high water activity, and presence of autolytic enzymes [2]. As one of the most important food fish in China, only alive crucian carp fish is accepted in market [4]. The spoilage of fish is a complicated process in which microbial, physical and chemical variations interact. Activity of the fish's own enzymes and chemical reactions are usually responsible for the initial loss of fish freshness, whereas the metabolic activities of microorganisms are involved in the whole spoilage [2]. Gutting of the fish immediately after capture can avoid the invasion of digestive tract proteases through the abdominal cavity to the tissue and prevent or slow degradation [3]. However, there may be chances of bacterial crosscontamination of fish during the gutting procedure. Microbial growth and metabolism are the major cause for food spoilage, the microbial populations may shift during storage and only a small fraction of fish microbiota is responsible for spoilage, known as "specific spoilage organisms" [5]. These specific spoilage organisms are present in low number in fresh fish and can eventually become dominant in spoilage microorganisms [6].

Bacterial flora isolated from eggs, skins, gills, and intestines have been described for some fish species. Bacteria recovered from the skin and gills may be transient rather than resident on the fish surfaces [7]. The gastrointestinal microbiota in fish is constituted of facultative and obligate anaerobes, which may vary among fish species with different digestive apparatus [8]. However, the zebrafish intestinal habitat select for specific bacterial taxa despite radical differences in host provenance and domestication status [9]. So far, the microbial community in fish guts has not been systematically characterized [10]. Most previous studies used traditional culture-dependent or DGGE, clone library methods to investigate the fish intestinal 
bacterial diversity [11-17]. With the development of the next-generation sequencing technologies, high-throughput sequencing platform is available for characterizing the bacterial community in the gut of fish [10,18-20]. More comprehensive information on fish gut microbiota would be obtained by highthroughput sequencing approaches. Illumina has fewer errors than 454 sequencing and it could provide a higher phylogenetic resolution than 454 based approaches [21]. The advantage of Illumina to provide 30 times more reads would enable us to perform in depth sequencing of samples in one run, making it an excellent tool for gut microbial diversity.

The objective of this study was to elucidate the bacterial and fungal flora in foreguts and hindguts of crucian carp. Specially, total DNAs of foreguts and hindguts were purified and analyzed by Illumina-based sequencing. The bacterial and fungal communities in foreguts and hindguts were further compared, the spoilage bacterial and fungal taxa in foreguts and hindguts were elucidated in the study.

\section{Material and Methods}

\section{Fish sample}

Ten live commercial-sized crucian carp with average weight of $250 \pm 20 \mathrm{~g}$ were purchased from aquatic market in March, 2015. They were kept alive before being processed. The fish were killed by slurry ice and gutted under sterile conditions. The portion of intestinal tract posterior from bile duct to the first distal loop (foreguts) and the intestinal tract anterior from anus to last anterior loop (hindguts) were removed for DNA extraction.

\section{DNA Extraction}

The foreguts (designated as QianC), hindguts (designated as HouC) were used for total DNA extraction. The total DNA was extracted using PowerSoil ${ }^{\circledR}$ DNA Isolation Kit (Mol Bio) according to the manufacture's instruction. Total DNA concentration and purity were monitored on $1 \%$ agarose gels.

\section{Amplicon Generation and Illumina MiSeq sequencing}

The primers S-D-Bact-0341-b-S-17 (5'- CCTACGGGNGGCWGCAG - 3') and S-D-Bact-0785-a-A-21 (5' - GACTACHVGGGTATCTAATCC - 3') targeting the V3-V4 hyper variable regions of bacterial 16S rRNA genes were selected for analyze bacterial taxa [22]. The primers ITS5-1737F: GGAAGTAAAAGTCGTAACAAGG; ITS2-2043R: GCTGCGTTCTTCATCGATGC targeting the ITS2 regions of fungal rRNA genes were adopted to analyze fungal taxa [23]. Both forward and reverse primers were tagged with adapter, pad and linker sequencing. Each barcode sequence was added to the reverse primer for pooling multiple samples into one run of sequencing. All PCR reactions were performed in a total volume of $30 \mu \mathrm{L}$ containing $15 \mu \mathrm{L}$ Phusion ${ }^{\circledR}$ High-Fidelity PCR Master Mix (New England Biolabs) and 0.5 units of AccuPrimer TM Taq DNA Polymerase (Life Technologies, USA), 0.2 $\mu \mathrm{M}$ of forward and reverse primers, and 10 ng template DNA. Thermal cycling conditions were as follows: an initial denaturation at $98^{\circ} \mathrm{C}$ for $1 \mathrm{~min}$, each of 30 cycles at $98^{\circ} \mathrm{C}$ for $10 \mathrm{~s}, 50^{\circ} \mathrm{C}$ for $30 \mathrm{~s}$, and $72^{\circ} \mathrm{C}$ for $60 \mathrm{~s}$, with a final extension at $72{ }^{\circ} \mathrm{C}$ for $5 \mathrm{~min}$.
Following amplification, $2 \mu \mathrm{L}$ of PCR product was used to verify successful amplification by $2 \%$ agarose gel electrophoresis. The products of triplicate PCR reaction from one sample were combined and the pooled mixtures were purified with GeneJET Gel Extraction Kit (Thermo Scientific) and analyzed on an Agilent 2100 Bioanalyzer using High Sensitivity DNA Chips (Agilent Technologies, Germany) for size distribution. The sequencing libraries were generated using NEB Next ${ }^{\circledR}$ Ultra $^{\mathrm{TM}}$ DNA Library Prep Kit for Illumina (NEB, USA) following manufacturer's recommendations and index codes were added. The library quality was assessed on the Qubit@2.0 Fluorometer (Thermo Scientific) and Agilent Bioanalyzer 2100 system (Agilent Technologies, Germany). Finally, the library was sequenced on an Illumina MiSeq platform at Magigen biotechnology Co. Ltd, Guangzhou, China.

\section{Combination and data preprocessing}

Forward and reverse sequences were merged by overlapping paired-end reads using FLASH (V1.2.7, http://ccb.jhu.edu/ software/FLASH/) [24]. All sequence reads with the same tag were assigned to the same sample according to the unique barcodes (raw tags). The raw tags were further strictly filtered by previous methods (clean tags) [25] and the quality of clean tags were detected by Qiime (V1.7.0 http://qiime.org/index. html) [22]. The low quality tags were removed. The tags with chimera were detected and removed using UCHIME Algorithm (http://www.drive5.com/usearch/manual/uchime_algo.html) $[26,27]$. The effective sequences were then clustered into operational taxonomic units (OTU) at $97 \%$ sequence similarity using the UPARSE-OTU and UPARSE-OTUref algorithms of UPARSE software package (Uparse v7.0.1001 http://drive5.com/ uparse/), the indices of alpha diversity were calculated [28]. Finally, the RDP classifier was used to assign representative sequence to the microbial taxa [29]. Sequence data have been deposited in the National Center for Biotechnology Information (NCBI) Sequence Read Archive (SRA) under the accession number SRP062743.

\section{Statistical analysis}

Cluster analysis was preceded by principal component analysis (PCA) using the QIIME software package. QIIME calculates both weighted and unweighted unifrac distances, which are phylogenetic measures of beta diversity [30], the phylogenetic relations among different microbial taxa were further displayed by KRONA [31]. Alpha diversity indices Chao1, ACE, Shannon, Simpson and coverage were calculated to reflect the diversity and richness of the endophytic community in different samples [32].

\section{Results}

\section{Fungal and bacterial species richness and diversity}

After qualify filtering the raw reads, 100013 bacterial sequences remained with an average length of $440 \mathrm{bp}, 69227$ fungal sequences remained with an average length of $284 \mathrm{bp}$ (Table 1). The number of different bacterial OTUs at the $97 \%$ similarity level ranged from 207 to 229 per sample with an average of 221 OTU. The number of different fungal OTUs at the 
$97 \%$ similarity level ranged from 56 to 128 per sample with an average of 94 OTU. The alpha diversity indices (Shannon and Simpson) calculated from bacterial OTUs of different samples indicated that the foreguts contained more diverse bacteria than those in hindguts; nevertheless, the hindguts contained more diverse fungi than foreguts (Table 2).

Total 229 bacterial OTUs were detected in foreguts, 148 OTUs were still detected in hindguts (Figure 1). Contrary to bacteria, the hindguts contained more fungal OTU than foreguts, and 42 OTUs were detected in foreguts simultaneously (Figure 2).

\section{Fungal and bacterial composition and community struc- ture}

Bacterial representative sequences of each OTU were classified into the bacterial domain $(99.96 \%$ of the total data set), the Cetobacterium were the most dominant bacterial genus observed (27.5\%). Desulfovibrio were the second most abundant $(19.3 \%)$. Fungal representative sequences of each OTU were classified into fungi ( $100 \%$ of the total data set), the Alternaria were the most dominant genus (39.3\%). Emericella were the second most abundant $(6.9 \%)$. The dominant bacterial and fungal species were different in the different samples. The bacterial genera Cetobacterium (23.9\%), Desulfovibrio (9.3\%), Shewanella (8.5\%), Bacteroides (3.2\%), Coryrebacterium (2.8\%),
Acinetobacter (1.5\%) and Methyloversatilis (1.2\%) were the most detected bacterial genera in foreguts, the hindguts contained Cetobacterium (31.1\%), Desulfovibrio (29.3\%), Shewanella (2.0\%), Bacteroides (2.5\%), Mycobacterium (1.6\%) and Thiomonas $(1.3 \%)$ as the dominant bacterial genera (Figure 3). With the digestion of food from foreguts to hindguts, the dominant bacterial genera Shewanella and Bacteroides were still detected in hindguts, other bacterial genera Pseudomonas (0.8\%), Thiomonas (0.9\%), Staphylococcus (0.3\%), Dysgonomonas (0.4\%) and Streptomyces $(0.5 \%)$ were detected both in foreguts and hindguts. Dietzia (0.4\%), Deinococcus (0.2\%), Flavobacterium $(0.1 \%)$, Chryseobacterium (0.1\%), Anaerococcus (0.2\%), Neisseria $(0.1 \%)$, Escherichia $(0.1 \%)$ and Enhydrobacter (0.1\%) were detected in foreguts not in hindguts, whereas Gordonia $(0.1 \%)$ Propionicimonas $(0.1 \%)$ and Halothiobacillus $(0.2 \%)$ were only detected in hindguts (Figure 3).

For the dominant fungal genera in the hindguts, Alternaria (39.3\%), Emericella (6.9\%) and Cochliobolus $(2.0 \%)$ were the most detected fungal genera (Figure 4), the dominant fungal genera Emericella (6.9\%) and Cochliobolus (2.0\%) coexisted in foreguts and hindguts. The Zygosaccharomyces (0.1\%) were detected both in foreguts and hindguts. Nevertheless, Alternaia $(78.6 \%)$, Massarina (0.8\%) and Fusarium (0.2\%) were only detected in hindguts (Figure 4).

Table 1: The characteristics of effective tags from samples of foreguts (designated as QianC), hindguts (designated as HouC) of crucian carp

\begin{tabular}{|c|c|c|c|c|c|c|c|c|c|c|}
\multirow{2}{*}{ Samples } & \multicolumn{4}{|c|}{ V3-V4 tags* } & \multicolumn{3}{c}{ ITS2 tags* } \\
\cline { 2 - 9 } & Numbers & Total length (bp) & Max length (bp) & Min length(bp) & Numbers & Total length (bp) & Max length (bp) & Min length (bp) \\
\hline QianC & 52116 & 22993523 & 555 & 330 & 51430 & 15488478 \\
\hline HouC & 47897 & 21066530 & 570 & 346 & 17797 & 4198103 & 389 \\
\hline
\end{tabular}

*V3-V4: V3-V4 hyper variable regions of bacterial 16S rRNA genes. ITS2: internal transcribed spacer 2 regions of fungal rRNA genes

Table 2: The alpha diversity indices of bacterial and fungal OTUs from samples of foreguts (designated as QianC), hindguts (designated as HouC) of crucian carp.

\begin{tabular}{|c|c|c|c|c|c|c|c|c|c|c|}
\hline \multirow{2}{*}{ Samples } & \multicolumn{5}{|c|}{ Bacterial OTUs } & \multicolumn{5}{|c|}{ Fungal OTUs } \\
\hline & Chao 1* & ACE & Shannon & Simpson & Coverage & Chao1 & ACE & Shannon & Simpson & Coverage \\
\hline QianC & 229 & 229 & 4.542197 & 0.89408404 & 1 & 64.75 & 68.61305 & 1.302989 & 0.445011 & 0.999123 \\
\hline HouC & 211 & 209.089878 & 4.523819 & 0.881296355 & 0.999827772 & 130.5 & 131.7518 & 1.672886 & 0.375113 & 0.999415 \\
\hline
\end{tabular}

* Both Chaol and ACE described an estimate of the total number of phylotypes in a source environment, and Chaol is particularly appropriate for data sets in which most phylotypes are relatively rare in the community, ACE is appropriate for data sets in which some phylotypes occur more frequently. Both Shannon and Simpson index comprehensively reflect the richness and evenness of community, Shannon index is more sensitive to the richness of the community, and Simpson index is more sensitive to the evenness of the community. Coverage is a non-parametric estimator of the proportion of phylotypes in a library of infinite size that would be represented in a smaller library.

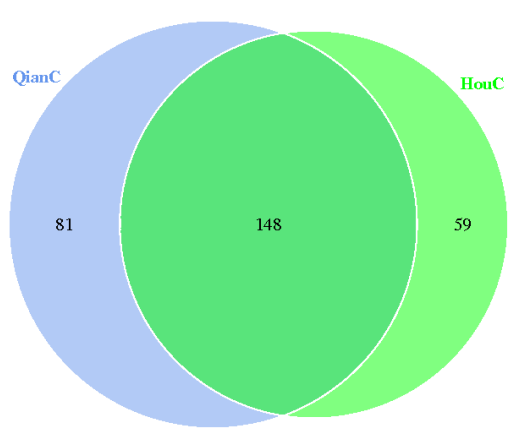

Figure 1: Venn diagrams of bacteria OTUs in samples of foregut (designated as QianC), hindgut (designated as HouC) of crucian carp.

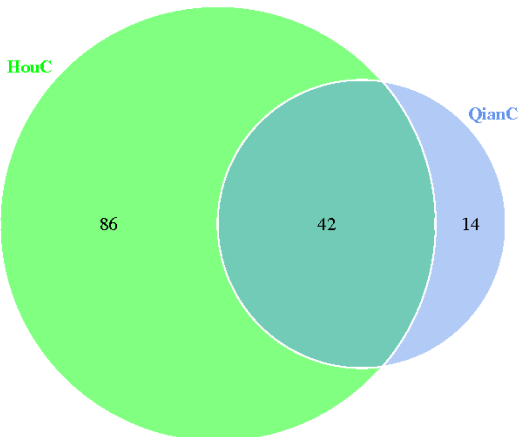

Figure 2: Venn diagrams of fungal OTUs in samples of foregut (designated as QianC), hindgut (designated as HouC) of crucian carp. 
The bacterial phyla Proteobacteria (55.6\%), Fusobacteria (27.5\%) and Bacteroidetes $(7.7 \%)$ in phylum level with high abundance were detected in two types of samples (Figure 5). The fungal phyla Ascomycota (50.4\%) and Basidiomycota(0.3\%) were detected in the two types of samples (Figure 6).

\section{Discussion}

Crucian carp is one of the most important food fish in China, and only alive fish is accepted in markets. Aquariums and tanks displaying live crucian carp caught in supermarkets and retail outlets are becoming increasingly common in China [4]. The water body in tanks is different from that in aquaculture, the effects of water body on intestinal flora of live crucian carp caught is still unknown. Our results illustrated that the foreguts contained more diverse bacteria than those in hindguts; nevertheless, the hindguts contained more diverse fungi than those in foreguts. The bacterial diversity distributed in foreguts and hindguts of crucian carp is consist with that in indigenous planktivorous gizzard shad, contratry to that in invasive Asian siler carp in Mississippi river basin, USA [10]. Although gut microbiota has become an integral component of the host, and received increasing attention [18], the fungal diversity in fish gut flora is not still reported [10]. The fish gut microbiota is only focused on bacteria [7-19]. Our results showed that the hindguts contained more diverse fungi than those in foreguts. The fungal phyla Ascomycota and Basidiomycota with high abundance were detected in guts of crucian carp. Fungal orders Pleosporales, Eurotiales, Saccharomycetales in foreguts were still detected in hindguts. The fungal taxa in foreguts were different from those in hindguts. The dominant fungal genera Alternaia $(78.6 \%)$, Massarina $(0.8 \%)$ and Fusarium $(0.2 \%)$ were only detected in hindguts. Most Alternaria species are saprophytes that are commonly found in soil or on decaying plant tissues. Some species are opportunistic plant pathogens that cause a range of disease (stem cancer, leaf blight, or leaf spot) with

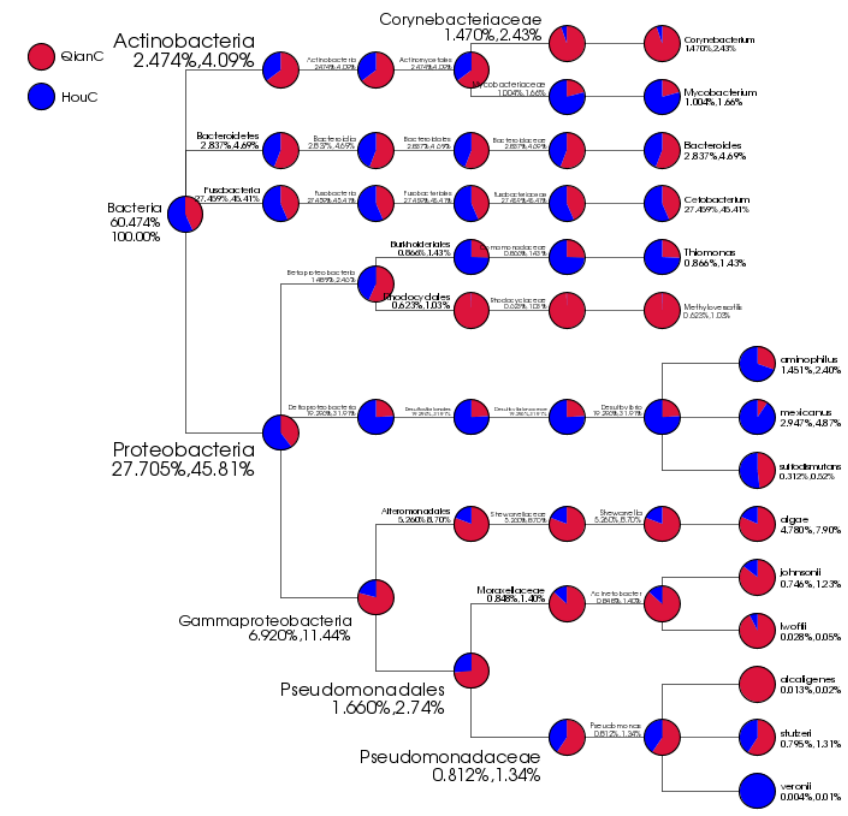

Figure 3: Phylogenetic tree of bacterial endophytic microbial communities in samples of foregut (designated as QianC), hindgut (designated as HouC) of crucian carp.

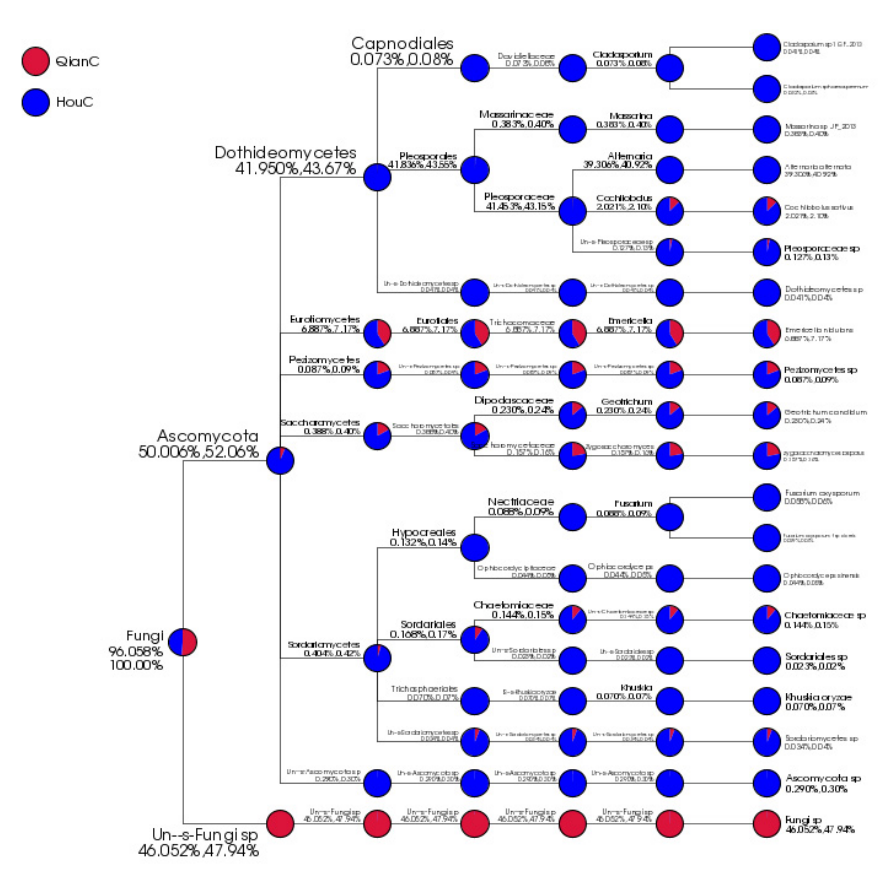

Figure 4: Phylogenetic tree of fungal endophytic microbial communities in samples of foregut (designated as QianC), hindgut (designated as HouC) of crucian carp.

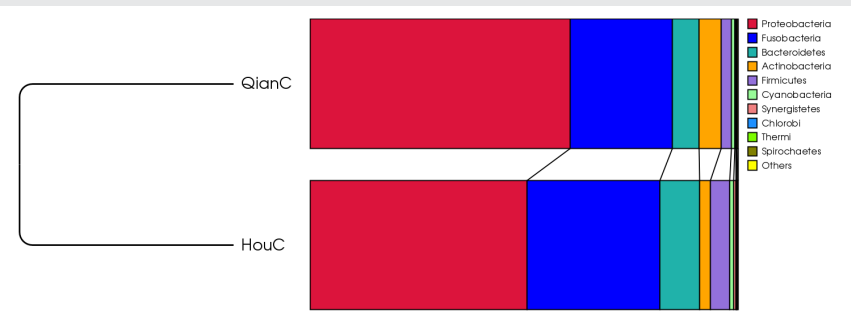

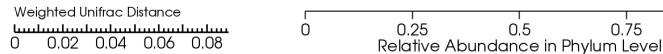

Figure 5: Relative abundance matrix in phylum level of bacterial endophytic microbial communities in samples of foregut (designated as QianC), hindgut (designated as HouC) of crucian carp.

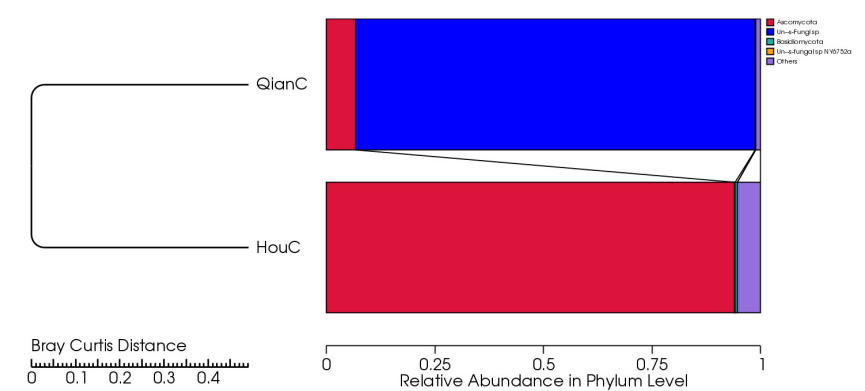

Figure 6: Relative abundance matrix in phylum level of fungal endophytic microbial communities in samples of foregut (designated as QianC), hindgut (designated as HouC) of crucian carp.

economic impact on the variety of important agronomic crops [33]. Alternaria species have been frequently isolated as endophytes from leaves and stems but not from roots [34-36]. Alternaria alternate has been isolated as the most dominant species in leaves [35]. So it was considered that the Alternaria, Emericella, and Cochliobolus might derive from the diets of crucian carp. 
More diverse bacteria in foreguts were detected than those in hindguts. The anaerobic bacterial genera Cetobacterium Desulfovibrio and Shewanella in foreguts were still detected in hindguts. The Cetobacterium spp. had been detected in the intestinal contents of goldfish (Carassius auratus) [8]. The Shewanella also been found in intestinal contents of goldfish, yellow catfish, rainbow rout $[8,11,17]$. The sulphate-reducing bacteria Desulfovibrio had not been detected in fish guts [7-19), although the Desulfovibrio are the most routinely recovered from animal and human faeces [37]. The results illustrated that the intestinal flora varied after the live crucian carp was caught and maintained in the tanks. The Desulfovibrio served as terminal oxidizers in the anaerobic degradation of organic matter entering the gastrointestinal tract and reduce sulphur and suphur-containing compounds to hydrogen sulphide $\left(\mathrm{H}_{2} \mathrm{~S}\right)$ [37]. The production of $\mathrm{H}_{2} \mathrm{~S}$ by the Desulfovibrio is potentially a major toxin to the fish gut epithelium and promoted the fish spoilage processes. The activities of Desulfovibrio might be responsible for the changes in odour, flavor and texture of the fish meat. Our results were consistent with the fact that fresh fish spoilage can be very rapid after it is caught [3]. So the manipulation of intestinal flora to preserve fresh crucian carp in tank would be further studied.

\section{References}

1. Xiao J, Zou T, Chen Y, Chen L, Liu S, et al (2011) Coexistence of diploid, triploid and treraploid crucian carp (Carassius auratus) in natural waters. 12:20. Link: https://goo.gl/20KjTi

2. Li T, Li J, Hu W, Zhang X, Li X, et al. (2012) Shelf-life extension of crucian carp (Carassius auratus) using natural preservatives during chilled storage. Food Chemistry 135:140-145. Link: https://goo.gl/QoBnUA

3. Ghaly AE, Dave D, Budge S, Brooks MS (2010) Fish spoilage mechanisms and preservation techniques: review. American Journal of Applied Sciences 7: 859-877. Link: https://goo.gl/6ablrG

4. Zeng P, Chen T, Shen J (2014) Effects of cold acclimation and storage temperature on crucian carp (Carassius auratu gibelios) in a waterless preservation. Fish Physiology and Biochemistry 40: 973-982. Link: https://goo.gl/1AZBWy

5. Wang $H$, Luo $Y$, Huang $H, X u Q$ (2014) Microbial succession of grass carp (Ctenopharyngodon idellus) filets during storage at $4{ }^{\circ} \mathrm{C}$ and its contribution to biogenic amines' formation. Int J Food Microbiol 190: 66-71. Link: https://goo.gl/xOZS5Y

6. Zhang Y1, Li Q1, Li D1, Liu X1, Luo Y (2015) Changes in the microbia communities of air-packaged and vacuum-packaged common carp (Cyprinus carpio) stored at $4{ }^{\circ} \mathrm{C}$. Food Microbiology 52: 197-204. Link: https://goo.gl/6QWiSA

7. Cahill MM (1990) Bacterial flora of fishes: a review. Microbial Eology 19: 2141. Link: https://goo.gl/9erL1x

8. Silva FC1, Nicoli JR, Zambonino-Infante JL, Kaushik S, Gatesoupe FJ (2011) Influence of the diet on the microbial diversity of faecal and gastrointestinal contents in gilthead sea bream (Sparus aurata) and intestinal contents in goldfish (Carassius auratus). FEMS Microbiology Ecology 78: 285-296. Link: https://goo.gl/uXOmHo

9. Roeselers G1, Mittge EK, Stephens WZ, Parichy DM, Cavanaugh CM, et al (2011) Evidence for a core gut microbiota in the zebrafish. ISME J 5: 15951608. Link: https://goo.gl/Z8PZYp

10. Ye L, Amberg J, Chapman D, Gaikowski M, Liu WT (2016) Fish gut microbiota analysis differentiates physiology and behavior of invasive Asian carp and indigenous American fish. ISME J 8: 2076. Link: https://goo.gl/2BdoWu

11. Huber I, Spanggaard B, Appel KF, Rossen L, Nielsen T, et al (2004) Phylogenetic analysis and in situ identification of the intestinal microbial community of rainbow trout (Oncorhynchus mykiss, Walbaum). J Appl Microbiol 96: 117132. Link: https://goo.gl/SvkeSr

12. Al-Harbi AH, Uddin N (2005) Bacterial diversity of tilapia (Oreochromis niloticus) cultured in brackish water in Saudi Arabia. Aquaculture 250: 566572. Link: https://goo.gl/Qkr779

13. Pond MJ, Stone DM, Alderman DJ (2006) Comparison of conventional and molecular techniques to investugate the intestinal microflora of rainbow trout (Oncorhynchus mykiss). Aquaculture 261: 194-203. Link: https://goo.gl/LRgSaj

14. Ward NL1, Steven B, Penn K, Methé BA, Detrich WH 3rd. (2009) Characterization of the intestinal microbiota of two antarctic notothenioid fish species. Extremophiles 1 3: 679-685. Link: https://goo.gl/5ShtMI

15. Han S, Liu Y, Zhou Z, He S, Cao Y, et al. (2010) Analysis of bacteria dicersity in the intestine of grass carp (Ctenopharyngodon idellus) based on 16S rDNA gene sequences. Aquaculture Research 42: 47-56. Link: https://goo.gl/DHFPVw

16. Tapia-Paniagua ST1, Chabrillón M, Díaz-Rosales $P$, de la Banda IG, Lobo C, et al. (2010) Intestinal microbiota diversity of the flat fish Solea senegalensis (Kaup, 1858) following probiotic administration. Microb Ecol 60: 310-319. Link: https://goo.gl/JBF5jb

17. Wu S, Gao T, Zheng Y, Wang W, Cheng Y, et al. (2010) Microbial diveristy of intestinal contents and mucus in yellow catfish (Pelteobagrus fulvidraco). Aquaculture 303: 1-7. Link: https://goo.gl/dh4nRw

18. Wu S, Wang G, Angert ER, Wang W, Li W, et al. (2012) Composition, diversity, and origin of the bacterial community in grass carp intestine. Plos one 7 e30440. Link: https://goo.gl/gUiHCS

19. Carda-Dieguez M, Mira A, Fouz B (2013) Pyrosequencing survey of intestinal microbiota diversity in cultured sea bass (Dicentrarchus labrax) fed functional diets. FEMS Microbiology Ecology 87: 451-459. Link: https://goo.gl/TJMvXa

20. Rungrassamee W1, Klanchui A1, Maibunkaew S1, Chaiyapechara S2 Jiravanichpaisal P, et al. (2014) Characterization of intestinal bacteria in wild and domesticated adult black tiger shrimp (Penaeus monodon). Plos one 9: e91853. Link: https://goo.gl/AkKqbi

21. Shi Y1, Yang H, Zhang T, Sun J, Lou K (2014) Illumina-based analysis of endophytic bacterial diversity and space-time dynamics in sugar beet on the north slope of Tianshan mountain. Appl Microbiol Biotechnol 98: 6375-6385. Link: https://goo.gl/3PhHqf

22. Caporaso JG1, Lauber CL, Walters WA, Berg-Lyons D, Huntley J, et al (2012) Ultra-high-throughput microbial community analysis on the Illumina HiSeq and MiSeq platforms. ISME J 6: 1621-1624. Link: https://goo.gl/yNpnrl

23. Degnan PH, Ochman H (2012) Illumina-based analysis of microbial community diversity. ISME J 6: 183-194. Link: https://goo.gl/423p1X

24. Magoč T1, Salzberg SL (2011) FLASH: fast length adjustment of short reads to improve genome assemblies. Bioinformatics 27: 2957-2963. Link: https://goo.gl/rT3bPJ

25. Bokulich NA1, Subramanian S, Faith JJ, Gevers D, Gordon JI, et al. (2013) Quality-filtering vastly improves diversity estimates from illumine amplicon sequencing. Nature Methods 10: 57-59. Link: https://goo.gl/r5tHmA

26. Edgar RC1, Haas BJ, Clemente JC, Quince C, Knight R (2011) UCHIME improves sensitivity and speed of chimera detection. Bioinformatics 27 2194-2200. Link: https://goo.gl/G6teX1

27. Haas BJ, Gevers D, Earl AM, Feldgarden M, Ward DV, et al. (2011) Chimeric 16S 
rRNA sequence formation and detection in Sanger and 454-pyrosequenced PCR amplicons. Genome Research 21: 494-504. Link: https://goo.gl/My4D1Q

28. Edgar RC (2013) UPARSE: highly accurate OTU sequences from microbial amplicon reads. Nature methods 10: 996-998. Link: https://goo.gl/2PbhjK

29. Wang Q1, Garrity GM, Tiedje JM, Cole JR (2007) Naïve Bayesian classifier for rapid assignment of rRNA sequences into the new bacterial taxonomy. Appl Environ Microbiol 73: 5261-5267. Link: https://goo.gl/mHqXCA

30. Kõljalg U, Nilsson RH, Abarenkov K, Tedersoo L, Taylor AFS, et al. (2013) Towards a unified paradigm for sequence-based identification of fungi. Molecular Ecology 22: 5271-5277. Link: https://goo.gl/SMkO2D

31. Ondov BD, Bergman NH, Phillippy AM (2011) Interactive metagenomic visualization in a Web browser. BMC bioinformatics 12: 385-394. Link: https://goo.gl/IOXHCm

32. Kemp PF, Aller JY (2004) Bacterial diversity in aquatic and other environments: what 16S rDNA libraries can tell us. FEMS Microbiol Ecol 47: 161-177.1 Link: https://goo.gl/asR4N8
33. Thomma BP (2003) Alternaria spp.: from general saprophyte to specific parasite. Mol Plant Pathol 4: 225-236. Link: https://goo.gl/e2Ac2f

34. Morita S, Azuma M, Aoba T, Satou H, Narisawa K, et al. (2003) Induced systemic resistance of Chinese cabbage to bacterial leaf spot and Alternaria leaf spot by the root endophytic fungus, Heteroconium chaetospira. J Gen Plant Pathol 69: 71-75. Link: https://goo.gl/sWxqt2

35. Guo LD, Xu L, Zheng WH, Hyde KD (2004) Genetic variation of Alternaria alternate, an endophytic fungus isolated from Pinus tabulaeformis as determined by random amplified microsatellites (RAMS). Fungal Diversity 16: 53-65. Link: https://goo.gl/Hs9JyX

36. Gu W (2009) Bioactive metabolites from Alternaria brassicicola ML-P08, an endophytic fungus residing in Malus halliana. World $\mathrm{J}$ Microbiol Biotechnol 25: 1677-1683. Link: https://goo.gl/OYkP7Y

37. Scanlan PD1, Shanahan F, Marchesi JR (2009) Cuture-independent analysis of desulfovibrios in the human distal colon of healthy, colorectal cancer and polypectomized individuals. FEMS Microbiol Ecol 69: 213-221. Link: https://goo.gl/H4gxfBCm

Copyright: (C) 2017 Wang M, et al. This is an open-access article distributed under the terms of the Creative Commons Attribution License, which permits unrestricted use, distribution, and r eproduction in any medium, provided the original author and source are credited. 